\title{
Uric Acid Urolithiasis
}

National Cancer Institute

\section{Source}

National Cancer Institute. Uric Acid Urolithiasis. NCI Thesaurus. Code C123245.

Urolithiasis in which the composition of the stones is predominantly urate. 